\title{
Stochastic Volterra Equation Driven by Wiener Process and Fractional Brownian Motion
}

\author{
Zhi Wang ${ }^{1,2}$ and Litan Yan ${ }^{1}$ \\ ${ }^{1}$ Department of Mathematics, College of Science, Donghua University, 2999 North Renmin Road, Songjiang, Shanghai 201620, China \\ ${ }^{2}$ School of Sciences, Ningbo University of Technology, 201 Fenghua Road, Ningbo 315211, China
}

Correspondence should be addressed to Litan Yan; litanyan@hotmail.com

Received 6 September 2013; Accepted 14 October 2013

Academic Editor: Yaozhong $\mathrm{Hu}$

Copyright (C) 2013 Z. Wang and L. Yan. This is an open access article distributed under the Creative Commons Attribution License, which permits unrestricted use, distribution, and reproduction in any medium, provided the original work is properly cited.

For a mixed stochastic Volterra equation driven by Wiener process and fractional Brownian motion with Hurst parameter $H>1 / 2$, we prove an existence and uniqueness result for this equation under suitable assumptions.

\section{Introduction}

In recent years, there has been considerable interest in studying fractional Brownian motion ( $\mathrm{fBm}$ ) due to some of its compact properties such as self-similarity, stationary increments, Hölder's continuity, and long-range dependence (when $H>1 / 2$ ), and also due to its applications in various scientific areas including telecommunications, turbulence, image processing, finance, and other fields. It is a suitable generalization of standard Wiener process. Some surveys and complete literatures could be found in Biagini et al. [1], Hu [2], Mishura [3], Nualart [4], and Zähle [5]. The so-called $\mathrm{fBm}$ with index $H \in(0,1)$ is a zero mean Gaussian process $B^{H}=\left\{B_{t}^{H}, t \geq 0\right\}$ with the covariance:

$$
E\left[B_{t}^{H} B_{s}^{H}\right]=\frac{1}{2}\left[s^{2 H}+t^{2 H}-|t-s|^{2 H}\right]
$$

Since $B^{H}$ is neither a semimartingale nor a Markov process unless $H=1 / 2$, the classical Itô theory is not available when dealing with it. There are several approaches to define an integral with respect to fBm. One possibility is Skorokhod or divergence integral introduced in the fractional Brownian setting in Decreusefond and Üstünel [6]. We will use a pathwise integral, defined first in Zähle [5], for fBm with
$H>1 / 2$ as a Young integral. The aim of this paper is to study the following mixed stochastic Volterra equation on $\mathbb{R}^{d}$ :

$$
\begin{aligned}
X_{t}= & X_{0}+\int_{0}^{t} a\left(t, s, X_{s}\right) d s+\int_{0}^{t} b\left(t, s, X_{s}\right) d W_{s} \\
& +\int_{0}^{t} c\left(t, s, X_{s}\right) d B_{s}^{H}, \quad t \in[0, T],
\end{aligned}
$$

where $a=\left(a^{i}\right)_{d \times 1}:[0, T]^{2} \times \mathbb{R}^{d} \rightarrow \mathbb{R}^{d}, b=\left(b^{i, j}\right)_{d \times r}:[0, T]^{2} \times$ $\mathbb{R}^{d} \rightarrow \mathbb{R}^{d} \times \mathbb{R}^{r}, c=\left(c^{i, j}\right)_{d \times m}:[0, T]^{2} \times \mathbb{R}^{d} \rightarrow \mathbb{R}^{d} \times \mathbb{R}^{m}, W$ is an $r$-dimensional standard Wiener process, and $B^{H}$ is an $m$ dimensional $\mathrm{fBm}$ with $H>1 / 2$. We assume that the processes $W$ and $B^{H}$ are independent, the integral with respect to $W$ is Itô type, and the integral with respect to $B^{H}$ is a pathwise Riemann-Stieltjes integral in the sense of Zähle [5].

The mixed stochastic differential equation:

$$
\begin{aligned}
X_{t}= & X_{0}+\int_{0}^{t} a\left(s, X_{s}\right) d s+\int_{0}^{t} b\left(s, X_{s}\right) d W_{s} \\
& +\int_{0}^{t} c\left(s, X_{s}\right) d B_{s}^{H}, \quad t \in[0, T]
\end{aligned}
$$

was first considered in Kubilius [7], where unique solvability was proved in one-dimensional case for time-independent coefficients and zero drift; that is, $a=0$. Later, in Zähle [8], existence of a solution to (3) was proved under less 
restrictive assumptions, but only locally, that is, up to a random time. In Guerra and Nualart [9], global existence and uniqueness of solution to (3) was established under the assumption that $W$ and $B^{H}$ are independent. The latter result was obtained in Mishura and Shevchenko [10, 11] without the independence assumptions, and it was also shown in Mishura and Shevchenko [11] that all moments of the solution are finite for $H>3 / 4$. The motivation to consider such equations comes from some financial applications, where Wiener process as a model is inappropriate because of the lack of memory, and $\mathrm{fBm}$ with $H>1 / 2$ is too smooth. A model driven by both processes is free of such drawbacks. Stochastic Volterra equations are also studied by many authors (see, e.g., [12-16]). In this paper, we consider the mixed stochastic Volterra equations driven by both Wiener process and $\mathrm{fBm}$, and also give an existence and uniqueness result for this equation under suitable assumptions.

This paper is organized as follows. Section 2 contains some preliminaries on function spaces and lists our assumptions on the coefficients of (2). It also states the main results in this section. In Section 3, we will give some estimates of fractional and Itô integrals, based on the fractional calculus. Finally, in Section 4, we will prove Theorem 1 using the classical Yamada and Watanabe theorem $[17,18]$ to check the pathwise uniqueness property and the existence of weak solutions of (2).

\section{Preliminaries and Main Results}

Let $(\Omega, \mathscr{F}, P)$ be a complete probability space equipped with a filtration satisfying standard assumptions. Denote $B^{H}=$ $\left\{B_{t}^{H}, t \in[0, T]\right\}$ an $m$-dimensional $\mathrm{fBm}$ with $H>1 / 2$ and $W=\left\{W_{t}, t \in[0, T]\right\}$ an $r$-dimensional standard Wiener process, independent of $B^{H} . X_{0}$ is a $d$-dimensional random variable independent of $\left(B^{H}, W\right)$. For each $t \in[0, T]$, we denote by $\mathscr{F}_{t}$ the $\sigma$-field generated by the random variables $\left\{X_{0}, B_{s}^{H}, W_{s}, s \in[0, t]\right\}$ and the $P$-null sets. $\left\{\mathscr{G}_{t}, t \in[0, T]\right\}$ denotes the bigger filtrations such that $\left\{\mathscr{G}_{t}\right\}$ is right-continuous and $\mathscr{G}_{0}$ contains the $P$-null sets, $X_{0}, B^{H}$ are $\mathscr{G}_{0^{-}}$ measurable, and $W$ is a $\mathscr{G}_{t}$-Wiener process.

Let us now introduce some function spaces for later use. Leting $1 / 2<H<1$ and $\alpha \in(1-H, 1 / 2)$, for any measurable function $f:[0, T] \rightarrow \mathbb{R}^{d}$, we introduce the following notation:

$$
\|f(t)\|_{\alpha}:=|f(t)|+\int_{0}^{t} \frac{|f(t)-f(s)|}{(t-s)^{\alpha+1}} d s .
$$

Denote by $W_{0}^{\alpha, \infty}$ the space of measurable function $f$ : $[0, T] \rightarrow \mathbb{R}^{d}$ such that

$$
\|f\|_{\alpha, \infty}:=\sup _{t \in[0, T]}\|f(t)\|_{\alpha}<\infty .
$$

For any $0<\lambda \leq 1$, let $C^{\lambda}$ be space of $\lambda$-Hölder continuous functions $f:[0, T] \rightarrow \mathbb{R}^{d}$ such that

$$
\|f\|_{\lambda}:=\|f\|_{\infty}+\sup _{0 \leq s<t \leq T} \frac{|f(t)-f(s)|}{(t-s)^{\lambda}}<\infty,
$$

where $\|f\|_{\infty}:=\sup _{t \in[0, T]}|f(t)|$. Given any $0<\varepsilon<\alpha$, we have the following inclusions:

$$
C^{\alpha+\varepsilon} \subset W_{0}^{\alpha, \infty} \subset C^{\alpha-\varepsilon} \text {. }
$$

Notice that both the $\mathrm{fBm} B^{H}$ with $H>1 / 2$ and the standard Wiener process $W$ have their trajectories in $W_{0}^{\alpha, \infty}$.

In what follows, we will assume the following standard hypotheses. Throughout the paper, the symbol $C$ will denote a generic constant, whose value is not significant and can change from one line to another. To emphasize its dependence on some parameters, we will put them into subscripts consider the following.

$\left(H_{1}\right) a:[0, T]^{2} \times \mathbb{R}^{d} \rightarrow \mathbb{R}^{d}$ is a measurable function such that there exists $a_{0} \in L^{\rho}\left([0, T]^{2} ; \mathbb{R}^{d}\right)$ with $\rho \geq 2,0<$ $\mu \leq 1$ and for all $N \geq 0$ there exists $C>0$ such that

$$
\begin{gathered}
|a(t, s, x)-a(t, s, y)| \leq C|x-y|, \\
\left|a\left(t_{1}, s, x\right)-a\left(t_{2}, s, x\right)\right| \leq C\left|t_{1}-t_{2}\right|^{\mu}, \\
|a(t, s, x)| \leq C(1+|x|), \\
\left|a\left(t_{1}, s, x_{1}\right)-a\left(t_{1}, s, x_{2}\right)-a\left(t_{2}, s, x_{1}\right)+a\left(t_{2}, s, x_{2}\right)\right| \\
\leq C\left|t_{1}-t_{2}\right|\left|x_{1}-x_{2}\right|, \\
\text { for all } x, y, x_{1}, x_{2} \in \mathbb{R}^{d} \text { and } s, t, t_{1}, t_{2} \in[0, T] .
\end{gathered}
$$

$\left(H_{2}\right) b:[0, T]^{2} \times \mathbb{R}^{d} \rightarrow \mathbb{R}^{d} \times \mathbb{R}^{m}$ is a measurable function and there exists a constant $C>0$ such that

$$
\begin{gathered}
|b(t, s, x)-b(t, s, y)| \leq C|x-y|, \\
|b(t, s, x)| \leq C(1+|x|),
\end{gathered}
$$

for all $x, y \in \mathbb{R}^{d}$ and $s, t \in[0, T]$.

$\left(H_{3}\right) c:[0, T]^{2} \times \mathbb{R}^{d} \rightarrow \mathbb{R}^{d} \times \mathbb{R}^{m}$ is a measurable function. Moreover, there exist the derivatives $\partial_{x} c(t, s, x)$, $\partial_{t} c(t, s, x), \partial_{x, c}^{2} c(t, s, x)$ and constants $0<\beta, \mu, \delta \leq 1$ and $C>0$ such that the following properties hold:

$$
\begin{aligned}
& |c(t, s, x)-c(t, s, y)| \\
& \quad+\left|\partial_{t} c(t, s, x)-\partial_{t} c(t, s, y)\right| \leq C|x-y|, \\
& \left|\partial_{x_{i}} c(t, s, x)-\partial_{y_{i}} c(t, s, y)\right| \\
& \quad+\left|\partial_{x_{i}, t}^{2} c(t, s, x)-\partial_{y_{i}, t}^{2} c(t, s, y)\right| \leq C|x-y|^{\delta}, \\
& \left|c\left(t_{1}, s, x\right)-c\left(t_{2}, s, x\right)\right| \\
& \quad+\left|\partial_{x_{i}} c\left(t_{1}, s, x\right)-\partial_{x_{i}} c\left(t_{2}, s, x\right)\right| \leq C\left|t_{1}-t_{2}\right|^{\mu}, \\
& \quad c\left(t, s_{1}, x\right)-c\left(t, s_{2}, x\right) \mid \\
& \quad+\left|\partial_{t} c\left(t, s_{1}, x\right)-\partial_{t} c\left(t, s_{2}, x\right)\right| \leq C\left|s_{1}-s_{2}\right|^{\beta}, \\
& \left|\partial_{x_{i}, t}^{2} c\left(t, s_{1}, x\right)-\partial_{x_{i}, t}^{2} c\left(t, s_{2}, x\right)\right| \\
& \quad+\left|\partial_{x_{i}} c\left(t, s_{1}, x\right)-\partial_{x_{i}} c\left(t, s_{2}, x\right)\right| \leq C\left|s_{1}-s_{2}\right|^{\beta},
\end{aligned}
$$


for all $x, y \in \mathbb{R}^{d}, s, t, t_{1}, t_{2}, s_{1}, s_{2} \in[0, T], i=1$, $2, \ldots, d$.

Denote by $E^{W}$ the conditional expectation given $\mathscr{F}_{0}$, that is, given $X_{0}$ and $B^{H}$. A strong solution of (2) is a $d$ dimensional $\mathscr{F}_{t}$-adapted stochastic process $\left\{X_{t}, t \in[0, T]\right\}$ such that a.s. the trajectories of $X$ belong to $W_{0}^{\alpha, \infty}$ and $\int_{0}^{T} E^{W}\left\|X_{s}\right\|_{\alpha}^{2} d s<\infty$, which satisfies (2) a.s. The main result of our paper is the following theorem on the existence and uniqueness of a solution for (2).

Theorem 1. Suppose that the coefficients $a, b$, and $c$ satisfy the assumptions $\left(H_{1}\right),\left(H_{2}\right)$, and $\left(H_{3}\right)$ with $\beta>1-H, \delta>1 / H-$ $1, \min \{\beta, \delta /(\delta+1)\}>1-\mu$, respectively. If $\max \{1-H, 1-\mu\}<$ $\alpha<\min \{1 / 2, \beta, \delta /(\delta+1)\}$ and $\rho \leq 1 / \alpha$, then (2) has a unique strong solution $X$.

Notice that in all our results we can replace the $\mathrm{fBm} B^{H}$ with an arbitrary stochastic process with Hölder continuous trajectories of order $\lambda>1 / 2$.

\section{Integral Estimates}

In this section, we will first briefly recall some basic definitions of fractional integrals and derivatives and define the integral with respect to $\mathrm{fBm}$ as a generalized Stieltjes integral (see, e.g., Nualart and Răşcanu [19] and Zähle [5]). We will also give some estimates of this integral.

Let $f \in L^{1}(a, b)$ and let $0<\alpha<1$. Consider the left-sided and right-sided fractional Riemann-Liouville integrals of $f$ of order $\alpha$ :

$$
\begin{aligned}
& I_{a^{+}}^{\alpha} f(x):=\frac{1}{\Gamma(\alpha)} \int_{a}^{x} f(t)(x-t)^{\alpha-1} d t, \\
& I_{b^{-}}^{\alpha} f(x):=\frac{1}{\Gamma(\alpha)} \int_{x}^{b} f(t)(t-x)^{\alpha-1} d t
\end{aligned}
$$

if the integrals exist for almost all $x \in(a, b)$ respectively, where $\Gamma(\cdot)$ denotes the gamma function defined by

$$
\Gamma(z)=\int_{0}^{\infty} t^{z-1} e^{-t} d t, \quad z>0 .
$$

Let $I_{a^{+}}^{\alpha}\left(L^{p}\right)$ (resp., $\left.I_{b^{-}}^{\alpha}\left(L^{p}\right)\right)$ be the image of $L^{p}(a, b)$, by the operator $I_{a^{+}}^{\alpha}$ (resp., $I_{b^{-}}^{\alpha}$ ). If $f \in I_{a^{+}}^{\alpha}\left(L^{p}\right)$ (resp., $\left.I_{b^{-}}^{\alpha}\left(L^{p}\right)\right)$ then the Weyl derivatives of $f$ :

$$
\begin{aligned}
& D_{a^{+}}^{\alpha} f(x) \\
& :=\frac{1}{\Gamma(1-\alpha)}\left(\frac{f(x)}{(x-a)^{\alpha}}\right. \\
& \left.+\alpha \int_{a}^{x} \frac{f(x)-f(t)}{(x-t)^{\alpha+1}} d t\right) 1_{(a, b)}(x),
\end{aligned}
$$

$$
\begin{aligned}
& D_{b^{-}}^{\alpha} f(x) \\
& :=\frac{1}{\Gamma(1-\alpha)}\left(\frac{f(x)}{(b-x)^{\alpha}}\right. \\
& \left.+\alpha \int_{x}^{b} \frac{f(x)-f(t)}{(t-x)^{\alpha+1}} d t\right) 1_{(a, b)}(x)
\end{aligned}
$$

are defined for almost all $x \in(a, b)$ (the convergence of the integrals at the singularity $t=x$ holds pointwise for almost all $x \in(a, b)$ if $p=1$ and moreover in $L^{p}$-sense if $\left.1<p<\infty\right)$, respectively.

Let $f(a+):=\lim _{\varepsilon \succ 0} f(a+\varepsilon)$ and let $g(b-):=\lim _{\varepsilon \succ 0} g(b-$ $\varepsilon)$ (supposing that the limits exist and are finite) and define

$$
\begin{aligned}
& f_{a^{+}}(x):=(f(x)-f(a+)) 1_{(a, b)}(x), \\
& g_{b^{-}}(x):=(g(x)-g(b-)) 1_{(a, b)}(x) .
\end{aligned}
$$

Assuming that $f(a+), g(a+), g(b-)$ exists, $f_{a^{+}} \in I_{a^{+}}^{\alpha}\left(L^{p}\right)$ and $g_{b^{-}} \in I_{b^{-}}^{1-\alpha}\left(L^{p}\right)$ for some $p, q \geq 1,(1 / p)+(1 / q) \leq 1,0<\alpha<1$, the generalized Stieltjes Integral is defined as

$$
\begin{aligned}
\int_{a}^{b} f d g:= & (-1)^{\alpha} \int_{a}^{b} D_{a^{+}}^{\alpha} f_{a^{+}}(x) D_{b^{-}}^{1-\alpha} g_{b^{-}}(x) d x \\
& +f(a+)(g(b-)-g(a+)) .
\end{aligned}
$$

The following properties hold.

(i) If $\alpha p<1$, under the preceding assumptions we have $f \in I_{a^{+}}^{\alpha}\left(L^{p}\right)$ and we can write

$$
\int_{a}^{b} f d g:=(-1)^{\alpha} \int_{a}^{b} D_{a^{+}}^{\alpha} f_{a^{+}}(x) D_{b^{-}}^{1-\alpha} g_{b^{-}}(x) d x .
$$

(ii) If $f \in C^{\lambda}(a, b)$ and $g \in C^{\mu}(a, b)$ with $\lambda+\mu>1$, then the generalized Stieltjes integral exists; it is given by (16) and coincides with the Riemann-Stieltjes integral.

The linear spaces $I_{a^{+}}^{\alpha}\left(L^{p}\right)$ are Banach spaces with respect to the norms:

$$
\|f\|_{I_{a^{+}}^{\alpha}\left(L^{p}\right)}=\|f\|_{L^{p}}+\left\|D_{a^{+}}^{\alpha} f\right\|_{L^{p}} \sim\left\|D_{a^{+}}^{\alpha} f\right\|_{L^{p}},
$$

and the same is true for $I_{b^{-}}^{\alpha}\left(L^{p}\right)$. If $\alpha p<1$ then the norms on $I_{a^{+}}^{\alpha}\left(L^{p}\right)$ and $I_{b^{-}}^{\alpha}\left(L^{p}\right)$ are equivalent and if $a \leq c<d \leq b$, then

$$
\int_{c}^{d} f d g=\int_{a}^{b} 1_{(c, d)} f d g .
$$

Fix a parameter $0<\alpha<1 / 2$; denote by $W_{T}^{1-\alpha, \infty}$ the space of measurable functions $g:[0, T] \rightarrow \mathbb{R}$ such that

$$
\begin{aligned}
&\|g\|_{1-\alpha, \infty, T} \\
&:=\sup _{0<s<t<T}\left(\frac{|g(t)-g(s)|}{(t-s)^{1-\alpha}}\right. \\
&\left.\quad+\int_{s}^{t} \frac{|g(y)-g(s)|}{(y-s)^{2-\alpha}} d y\right)<\infty .
\end{aligned}
$$


Clearly,

$$
C^{1-\alpha+\varepsilon} \subset W_{T}^{1-\alpha, \infty} \subset C^{1-\alpha}
$$

for all $\varepsilon>0$. Moreover, if $g$ belongs to $W_{T}^{1-\alpha, \infty}$, we define

$$
\begin{aligned}
\Lambda_{\alpha}(g) & :=\frac{1}{\Gamma(1-\alpha)} \sup _{0<s<t<T}\left|\left(D_{t-}^{1-\alpha} g_{t-}\right)(s)\right| \\
& \leq \frac{1}{\Gamma(1-\alpha) \Gamma(\alpha)}\|g\|_{1-\alpha, \infty, T}<\infty
\end{aligned}
$$

We also denote by $W_{0}^{\alpha, 1}$ the space of measurable functions $f:[0, T] \rightarrow \mathbb{R}$ such that

$$
\begin{aligned}
\|f\|_{\alpha, 1}:= & \int_{0}^{T} \frac{|f(s)|}{s^{\alpha}} d s \\
& +\int_{0}^{T} \int_{0}^{s} \frac{|f(s)-f(y)|}{(s-y)^{1+\alpha}} d y d s<\infty .
\end{aligned}
$$

Then, if $f \in W_{0}^{\alpha, 1}$ and $g \in W_{T}^{1-\alpha, \infty}$, the integral $\int_{0}^{t} f(s) d g_{s}$ exists for all $t \in[0, T]$ and we have

$$
\int_{0}^{t} f(s) d g_{s}=\int_{0}^{T} f(s) 1_{(0, t)}(s) d g_{s}
$$

Furthermore, the following estimate holds:

$$
\left|\int_{0}^{t} f(s) d g_{s}\right| \leq \Lambda_{\alpha}(g)\|f\|_{\alpha, 1} .
$$

Given $f:[0, T]^{2} \rightarrow \mathbb{R}$ such that for any $t \in[0, T], f(t, \cdot) \in$ $W_{0}^{\alpha, 1}$, we can also consider the integral:

$$
G_{t}(f)=\int_{0}^{t} f(t, s) d g_{s}=\int_{0}^{T} f(t, s) 1_{(0, t)}(s) d g_{s}
$$

and the estimate:

$$
\left|\int_{0}^{t} f(t, s) d g_{s}\right| \leq \Lambda_{\alpha}(g)\|f(t, \cdot)\|_{\alpha, 1} .
$$

Now we will derive some useful estimates for the integrals involved in (2). Fix a parameter $\alpha \in(0,1 / 2)$. Consider first the ordinary Lesbesgue integral. Given $f:[0, T] \rightarrow \mathbb{R}^{d}$ a measurable function we define

$$
F_{t}^{a}(f)=\int_{0}^{t} a(t, s, f(s)) d s .
$$

Proposition 2. Assume that a satisfies $\left(H_{1}\right)$ with $\rho=1 / \alpha$ and $\mu>\alpha$. Let $f:[0, T] \rightarrow \mathbb{R}^{d}$ be a measurable function. If $f \in W_{0}^{\alpha, \infty}$, then $F^{a}(f) \in W_{0}^{\alpha, \infty}$, for all $t \in[0, T]$, one has

$$
\left\|F_{t}^{a}(f)\right\|_{\alpha} \leq C\left(\int_{0}^{t} \frac{|f(s)|}{(t-s)^{\alpha}} d s+1\right) .
$$

Proof. It follows from Proposition 2.1 in Besalú and Rovira [15] and the growth assumption in $\left(H_{1}\right)$ that

$$
\begin{aligned}
& \left|F_{t}^{a}(f)\right|+\int_{0}^{t} \frac{\left|F_{t}^{a}(f)-F_{s}^{a}(f)\right|}{(t-s)^{\alpha+1}} d s \\
& \leq C\left(\int_{0}^{t} \frac{|a(t, s, f(s))|}{(t-s)^{\alpha}} d s+1\right) \\
& \quad \leq C\left(\int_{0}^{t} \frac{1+|f(s)|}{(t-s)^{\alpha}} d s+1\right) \\
& \quad \leq C\left(\int_{0}^{t} \frac{|f(s)|}{(t-s)^{\alpha}} d s+1\right),
\end{aligned}
$$

as required.

Proposition 3. Assume that a satisfies $\left(H_{1}\right)$ with $P=1 / \alpha$ and $\mu>\alpha$. Let $f, h:[0, T] \rightarrow \mathbb{R}^{d}$ be measurable functions. If $f, h \in W_{0}^{\alpha, \infty}$, then for all $t \in[0, T]$, one has

$$
\left\|F_{t}^{a}(f)-F_{t}^{a}(h)\right\|_{\alpha} \leq C\left(\int_{0}^{t} \frac{\|f(s)-h(s)\|_{\alpha}}{(t-s)^{\alpha}} d s+1\right) .
$$

Proof. By Proposition 2.1 in Besalú and Rovira [15] and the growth assumption in $\left(H_{1}\right)$, one obtains

$$
\begin{aligned}
& \left\|F_{t}^{a}(f)-F_{t}^{a}(h)\right\|_{\alpha} \\
& \quad \leq C\left(\int_{0}^{t} \frac{|a(t, s, f(s))-a(t, s, h(s))|}{(t-s)^{\alpha}} d s+1\right) \\
& \quad \leq C\left(\int_{0}^{t} \frac{|f(s)-h(s)|}{(t-s)^{\alpha}} d s+1\right) \\
& \quad \leq C\left(\int_{0}^{t} \frac{\|f(s)-h(s)\|_{\alpha}}{(t-s)^{\alpha}} d s+1\right) .
\end{aligned}
$$
define

Given a measurable function $f:[0, T] \rightarrow \mathbb{R}^{d}$, let us

$$
G_{t}^{c}(f)=\int_{0}^{t} c(t, s, f(s)) d B_{s}^{H}
$$

Notice that if $f \in W_{0}^{\alpha, \infty}$, one has $c(t, \cdot, f(\cdot)) \in W_{0}^{\alpha, \infty}$.

Proposition 4. Assume that c satisfies $\left(\mathrm{H}_{3}\right)$ with $1-\mu<\alpha<$ $\beta, f \in W_{0}^{\alpha, \infty}$, then $G_{t}^{c}(f) \in W_{0}^{\alpha, \infty}$ for all $t \in[0, T]$ and we have

$$
\begin{aligned}
\left\|G_{t}^{c}(f)\right\|_{\alpha} \leq & C \Lambda_{\alpha}\left(B^{H}\right) \\
& \times \int_{0}^{t}\left((t-s)^{-2 \alpha}+s^{-\alpha}\right) \\
& \times\left(1+\|f(s)\|_{\alpha}\right) d s .
\end{aligned}
$$


Proof. It follows from Proposition 3.1 in Besalú and Rovira [15] and the growth assumption in $\left(\mathrm{H}_{3}\right)$ that

$$
\begin{aligned}
\left\|G_{t}^{c}(f)\right\|_{\alpha} \leq & C \Lambda_{\alpha}\left(B^{H}\right) \\
& \times\left(\int_{0}^{t}\left((t-s)^{-2 \alpha}+s^{-\alpha}\right)\right. \\
& \left.\times\|c(t, \cdot, f(\cdot))\|_{\alpha} d s+1\right) \\
\leq & C \Lambda_{\alpha}\left(B^{H}\right) \int_{0}^{t}\left((t-s)^{-2 \alpha}+s^{-\alpha}\right) \\
& \times\left(1+\|f(s)\|_{\alpha}\right) d s .
\end{aligned}
$$

Proposition 5. Assume thatc satisfies $\left(\mathrm{H}_{3}\right)$ with $1-\mu<\alpha<\beta$, $f, h \in W_{0}^{\alpha, \infty}$, then for all $t \in[0, T]$ one has

$$
\begin{aligned}
\left\|G_{t}^{c}(f)-G_{t}^{c}(h)\right\|_{\alpha} \leq C \Lambda_{\alpha}\left(B^{H}\right) \int_{0}^{t} & \left((t-s)^{-2 \alpha}+s^{-\alpha}\right) \\
& \times(1+\Delta f(s)+\Delta h(s)) \\
& \times\|f(s)-h(s)\|_{\alpha} d s
\end{aligned}
$$

where

$$
\Delta f=\sup _{s \in[0, T]} \int_{0}^{s} \frac{|f(s)-f(u)|^{\delta}}{(s-u)^{\alpha+1}} d u .
$$

Proof. Following Proposition 3.1 in Besalú and Rovira [15] and the assumptions in $\left(\mathrm{H}_{3}\right)$, we get that

$$
\begin{array}{r}
\left\|G_{t}^{c}(f)-G_{t}^{c}(h)\right\|_{\alpha} \\
\leq C \Lambda_{\alpha}\left(B^{H}\right)\left[\int_{0}^{t}\left((t-s)^{-2 \alpha}+s^{-\alpha}\right)\right. \\
\left.\times\|c(t, \cdot, f(\cdot))-c(t, \cdot, h(\cdot))\|_{\alpha} d s+1\right] \\
\times C \Lambda_{\alpha}\left(B^{H}\right)\left[\int_{0}^{t}\left((t-s)^{-2 \alpha}+s^{-\alpha}\right)\right. \\
\times(|c(t, s, f(s))-c(t, s, h(s))| \\
\quad+\int_{0}^{s}(\mid c(t, s, f(s))-c(t, s, h(s)) \\
\quad c(t, u, f(u))+c(t, u, h(u)) \mid \\
\left.\left.\left.\times\left((s-u)^{\alpha+1}\right)^{-1}\right) d u\right) d s+1\right] .
\end{array}
$$

By Lemma A.1 of Besalú and Rovira [15], the hypothesis $\left(H_{3}\right)$ imply that for all $x_{i}, \in \mathbb{R}, i=1, \ldots, 4, t, s_{1}, s_{2} \in[0, T]$, one has

$$
\begin{gathered}
\left|c\left(t, s_{1}, x_{1}\right)-c\left(t, s_{2}, x_{2}\right)-c\left(t, s_{1}, x_{3}\right)+c\left(t, s_{2}, x_{4}\right)\right| \\
\leq C\left(\left|x_{1}-x_{2}-x_{3}+x_{4}\right|+\left|x_{1}-x_{3}\right|\left|s_{2}-s_{1}\right|^{\beta}\right. \\
\left.+\left|x_{1}-x_{3}\right|\left(\left|x_{1}-x_{2}\right|^{\delta}+\left|x_{3}-x_{4}\right|^{\delta}\right)\right) .
\end{gathered}
$$

Thus, we obtain that

$$
\begin{aligned}
& \left\|G_{t}^{c}(f)-G_{t}^{c}(h)\right\|_{\alpha} \\
& \leq C \Lambda_{\alpha}\left(B^{H}\right)\left[\int_{0}^{t}\left((t-s)^{-2 \alpha}+s^{-\alpha}\right)(1+\Delta f(s)+\Delta h(s))\right. \\
& \times(|f(s)-h(s)| \\
& +\int_{0}^{s}(|f(s)-h(s)-f(u)+h(u)| \\
& \left.\left.\left.\times\left((s-u)^{\alpha+1}\right)^{-1}\right) d u\right) d s+1\right] \\
& \leq C \Lambda_{\alpha}\left(B^{H}\right) \int_{0}^{t}\left((t-s)^{-2 \alpha}+s^{-\alpha}\right)(1+\Delta f(s)+\Delta h(s)) \\
& \times\|f(s)-h(s)\|_{\alpha} d s .
\end{aligned}
$$

This completes the proof.

Finally, we will consider the estimate of Itô integral based on the Itô calculus. Let $f:[0, T] \rightarrow \mathbb{R}^{d}$ be a measurable function and define

$$
G_{t}^{b}(f)=\int_{0}^{t} b(t, s, f(s)) d W_{s}
$$

Proposition 6. Let $f:[0, T] \rightarrow \mathbb{R}^{d}$ be a $\mathscr{G}_{t}$-adapted stochastic process. If $f \in W_{0}^{\alpha, \infty}$ and $\int_{0}^{T} E^{W}\|f(s)\|_{\alpha}^{2} d s<\infty$ a.s., then for all $t \in[0, T]$ a.e., one has

$$
E^{W}\left\|G_{t}^{b}(f)\right\|_{\alpha}^{2} \leq C \int_{0}^{t}(t-s)^{-(1 / 2)-\alpha}\left[1+E^{W}\|f(s)\|_{\alpha}^{2}\right] d s .
$$

Proof. It follows from Lemma 3.7 in Guerra and Nualart [9] and the growth assumption in $\left(\mathrm{H}_{2}\right)$.

Proposition 7. Let $f, h:[0, T] \rightarrow \mathbb{R}^{d}$ be $\mathscr{G}_{t}$-adapted stochastic processes. If $f, h \in W_{0}^{\alpha, \infty}$ and $\int_{0}^{T} E^{W}\|\phi(s)\|_{\alpha}^{2} d s<\infty$, $\phi \in\{f, h\}$ a.s., then for all $t \in[0, T]$ a.e., one has

$$
\begin{aligned}
E^{W} & \left\|G_{t}^{b}(f)-G_{t}^{b}(h)\right\|_{\alpha}^{2} \\
& \leq C \int_{0}^{t}(t-s)^{-(1 / 2)-\alpha} E^{W}|f(s)-h(s)|^{2} d s .
\end{aligned}
$$


Proof. By Lemma 3.7 in Guerra and Nualart [9] and the Lipschitz assumption in $\left(\mathrm{H}_{2}\right)$, one has

$$
\begin{aligned}
& E^{W}\left\|G_{t}^{b}(f)-G_{t}^{b}(h)\right\|_{\alpha}^{2} \\
& \quad \leq C \int_{0}^{t}(t-s)^{-(1 / 2)-\alpha} E^{W}|b(t, s, f(s))-b(t, s, h(s))|^{2} d s \\
& \quad \leq C \int_{0}^{t}(t-s)^{-(1 / 2)-\alpha} E^{W}|f(s)-h(s)|^{2} d s,
\end{aligned}
$$

which completes the proof.

\section{Proof of the Theorem 1}

To prove Theorem 1, we will use the classical Yamada and Watanabe theorem [17, 18], which asserts that pathwise uniqueness and existence of weak solutions imply the existence of a strong solution. A weak solution of (2) in this paper is a triple $(\Omega, \mathscr{F}, P),\left(X, B^{H}, W\right),\left\{\mathscr{G}_{t}, t \in[0, T]\right\}$ such that:

(i) $(\Omega, \mathscr{F}, P)$ is a complete probability space, $\left\{\mathscr{G}_{t}\right\}$ is right-continuous, and $\mathscr{G}_{0}$ contains the P-null sets,

(ii) $B^{H}$ is a fBm which is $\mathscr{G}_{0}$-measurable,

(iii) $W$ is a $\mathscr{G}_{t}-r$-dimensional Wiener process,

(iv) $X$ is $\mathscr{G}_{t}$-adapted and has the trajectories belong to $W_{0}^{\alpha, \infty}$ and $\int_{0}^{T} E^{W}\left\|X_{s}\right\|_{\alpha}^{2} d s<\infty$ a.s,

(v) $\left(X, B^{H}, W\right)$ satisfies (2) a.s.

The pathwise uniqueness property holds for (2) means that if $\left(X, B^{H}, W\right)$ and $\left(Y, B^{H}, W\right)$ are two weak solutions, defined on the same probability space $(\Omega, \mathscr{F}, P)$ with the same filtration $\left\{\mathscr{G}_{t}\right\}$ and $X_{0}=Y_{0}$ a.s., then $X=Y$.

Let $f \in W_{0}^{\alpha, \infty}$. By Propositions 2.2 and 3.2 in Besalú and Rovira [15], the sample paths of the integral processes $G_{t}^{a}(f)$ and $G_{t}^{c}(f)$ are continuously differentiable and $\eta$ Hölder continuous of order $\eta<1-\alpha$, respectively. Therefore, if $X$ is a weak solution of (2), then the trajectories of $X$ are $\eta$-Hölder continuous for all $\eta<1 / 2$. Consequently, the pathwise uniqueness property holds for (2). It is exactly the same result as that of Theorem 4.4 given in Guerra and Nualart [9]. So it remains to prove that the existence of weak solutions for (2).

Let us now introduce the Euler approximation for (2). Take a sequence of partitions

$$
0=t_{0}^{n}<t_{1}^{n}<\cdots<t_{i}^{n}<\cdots<t_{n}^{n}=T
$$

of the interval $[0, T]$ such that

$$
\sup _{0 \leq i \leq n-1}\left|t_{i+1}^{n}-t_{i}^{n}\right| \longrightarrow 0
$$

as $n \rightarrow \infty$. Defined as $X_{t}^{0}=X_{0}$ and for all $n \geq 1$,

$$
\begin{aligned}
X_{t}^{n}= & X_{0}+\int_{0}^{t} a\left(t, v_{n}(s), X_{v_{n}(s)}^{n}\right) d s \\
& +\int_{0}^{t} b\left(t, v_{n}(s), X_{v_{n}(s)}^{n}\right) d W_{s} \\
& +\int_{0}^{t} c\left(t, v_{n}(s), X_{v_{n}(s)}^{n}\right) d B_{s}^{H},
\end{aligned}
$$

where $v_{n}(t)=\max \left\{t_{i}^{n}: t_{i}^{n} \leq t\right\}$. We first prove the tightness of the law of the sequence $\left\{X^{n}(t)\right\}$ in the space $C_{0}^{\eta}$ of $\eta$-Hölder continuous functions, with $\eta<1 / 2$, such that

$$
\lim _{\varepsilon \rightarrow 0} \sup _{0<|t-s|<\varepsilon} \frac{|f(t)-f(s)|}{(t-s)^{\eta}}=0 .
$$

Using the criterion given by Hamadouche [20], is sufficient to prove the following lemma.

Lemma 8. For any $N \geq 1$, there exists a random variable $R_{N}>0$, depending on $X_{0}$ and $B^{H}$, such that

$$
E^{W}\left[\left|X_{t}^{n}-X_{s}^{n}\right|^{2 N}\right] \leq R_{N}|t-s|^{N}
$$

for all $s, t \in[0, T]$ and $n \in \mathbb{N}$.

Proof. Firstly, we will show that there exists a random variable $K_{N}>0$ such that

$$
E^{W}\left\|X_{t}^{n}\right\|_{\alpha}^{2 N} \leq K_{N}
$$

for all $t \in[0, T]$ and $n \in \mathbb{N}$. In fact, from (46), we have

$$
\begin{gathered}
E^{W}\left\|X_{t}^{n}\right\|_{\alpha}^{2 N} \leq C_{N}\left\{\left|X_{0}\right|^{2 N}+E^{W}\left\|\int_{0}^{t} a\left(t, v_{n}(s), X_{v_{n}(s)}^{n}\right) d s\right\|_{\alpha}^{2 N}\right. \\
+E^{W}\left\|\int_{0}^{t} b\left(t, v_{n}(s), X_{v_{n}(s)}^{n}\right) d W_{s}\right\|_{\alpha}^{2 N} \\
\left.+E^{W}\left\|\int_{0}^{t} c\left(t, v_{n}(s), X_{v_{n}(s)}^{n}\right) d B_{s}^{H}\right\|_{\alpha}^{2 N}\right\} \\
:=C_{N}\left(\left|X_{0}\right|^{2 N}+A_{1}+A_{2}+A_{3}\right) .
\end{gathered}
$$

Using the estimate (28) and Hölder inequality, we get that

$$
\begin{aligned}
A_{1} & \leq C_{N} E^{W}\left(\int_{0}^{t} \frac{\left|X_{v_{n}(s)}^{n}\right|}{(t-s)^{\alpha}} d s+1\right)^{2 N} \\
& \leq C_{N} E^{W}\left(\int_{0}^{t}\left|X_{v_{n}(s)}^{n}\right|^{2} d s\right)^{N}+C_{N} \\
& \leq C_{N} E^{W}\left(\int_{0}^{t}\left|X_{v_{n}(s)}^{n}\right|^{2 N} d s\right)+C_{N} .
\end{aligned}
$$


Abstract and Applied Analysis

7

And also

$$
\begin{aligned}
A_{2} \leq & C_{N} E^{W}\left|\int_{0}^{t} b\left(t, v_{n}(s), X_{v_{n}(s)}^{n}\right) d W_{s}\right|^{2 N} \\
& +C_{N} E^{W}\left(\int_{0}^{t} \frac{\left|\int_{s}^{t} b\left(t, v_{n}(u), X_{v_{n}(s)}^{n}\right) d W_{u}\right|}{(t-s)^{\alpha+1}} d s\right)^{2 N} \\
:= & A_{21}+A_{22} .
\end{aligned}
$$

By Burkhölder-Davis-Gundy and Hölder inequalities, we obtain

$$
\begin{aligned}
A_{21} & \leq C_{N} E^{W}\left[\int_{0}^{t}\left|b\left(t, v_{n}(s), X_{v_{n}(s)}^{n}\right)\right|^{2 N} d s\right] \\
& \leq C_{N} \int_{0}^{t}\left[1+E^{W}\left|X_{v_{n}(s)}^{n}\right|^{2 N}\right] d s .
\end{aligned}
$$

Similarly, we get

$$
\begin{aligned}
A_{22} \leq & C_{N}\left(\int_{0}^{t} \frac{1}{(t-s)^{(2 N /(2 N-1))(\alpha+(1 / 2)-(((1 / 2)+\alpha) / 2 N))}} d s\right)^{2 N-1} \\
& \times E^{W}\left(\int_{0}^{t} \frac{\left|\int_{s}^{t} b\left(t, v_{n}(u), X_{v_{n}(s)}^{n}\right) d W_{u}\right|^{2 N}}{(t-s)^{\alpha+N+(1 / 2)}} d s\right) \\
\leq & C_{N} \int_{0}^{t}(t-s)^{-(3 / 2)-\alpha} \\
& \times E^{W}\left[\int_{s}^{t}\left|b\left(t, v_{n}(u), X_{v_{n}(s)}^{n}\right)\right|^{2 N} d u\right] d s .
\end{aligned}
$$

Now applying Fubini's theorem and using the growth assumption in $\left(\mathrm{H}_{2}\right)$, we have

$$
A_{22} \leq C_{N} \int_{0}^{t}(t-u)^{-(1 / 2)-\alpha}\left(1+E^{W}\left|X_{v_{n}(s)}^{n}\right|^{2 N}\right) d u .
$$

As a consequence,

$$
A_{2} \leq C_{N} \int_{0}^{t}(t-u)^{-(1 / 2)-\alpha} E^{W}\left|X_{v_{n}(s)}^{n}\right|^{2 N} d u+C_{N} .
$$

Further, applying the estimate (33) and Hölder inequality, we get

$$
\begin{aligned}
A_{3} \leq & C_{N} \Lambda_{\alpha}\left(B^{H}\right)^{2 N} \\
& \times E^{W}\left[\int_{0}^{t}\left((t-s)^{-2 \alpha}+s^{-\alpha}\right)\right. \\
& \left.\times\left(\left\|c\left(t, v_{n}(s), X_{v_{n}(s)}^{n}\right)\right\|_{\alpha}\right) d s\right]^{2 N} \\
\leq & C_{N} \Lambda_{\alpha}\left(B^{H}\right)^{2 N} \int_{0}^{t}\left((t-s)^{-2 \alpha}+s^{-\alpha}\right) \\
& \times\left[1+E^{W}\left\|X_{v_{n}(s)}^{n}\right\|_{\alpha}^{2 N}\right] d s .
\end{aligned}
$$

Combining these estimates, we have

$$
\begin{gathered}
E^{W}\left\|X_{t}^{n}\right\|_{\alpha}^{2 N} \leq C_{N}\left|X_{0}\right|^{2 N}+C_{N}\left(\Lambda_{\alpha}\left(B^{H}\right)^{2 N}+1\right) \\
\times \int_{0}^{t}\left((t-s)^{-(1 / 2)-\alpha}+s^{-\alpha}\right) \\
\times E^{W}\left\|X_{v_{n}(s)}^{n}\right\|_{\alpha}^{2 N} d s .
\end{gathered}
$$

Hence,

$$
\begin{aligned}
\sup _{0 \leq s \leq t} E^{W}\left\|X_{s}^{n}\right\|_{\alpha}^{2 N} \leq & C_{N}\left|X_{0}\right|^{2 N}+C_{N}\left(\Lambda_{\alpha}\left(B^{H}\right)^{2 N}+1\right) \\
& \times \int_{0}^{t}\left((t-s)^{-(1 / 2)-\alpha}+s^{-\alpha}\right) \\
& \times\left(\sup _{0 \leq u \leq s} E^{W}\left\|X_{u}^{n}\right\|_{\alpha}^{2 N}\right) d s .
\end{aligned}
$$

Therefore, by the generalized Gronwall lemma (Lemma 7.6 in Nualart and Răşcanu [19]), the estimate (49) is satisfied.

So it remains to prove that the estimate (48) is satisfied. Indeed, from (46), we have

$$
\begin{gathered}
E^{W}\left|X_{t}^{n}-X_{s}^{n}\right|^{2 N} \\
\leq C_{N}\left\{E^{W}\left|\int_{s}^{t} a\left(t, v_{n}(u), X_{v_{n}(s)}^{n}\right) d u\right|^{2 N}\right. \\
+E^{W}\left|\int_{s}^{t} b\left(t, v_{n}(u), X_{v_{n}(s)}^{n}\right) d W_{u}\right|^{2 N} \\
\left.+E^{W}\left|\int_{s}^{t} c\left(t, v_{n}(u), X_{v_{n}(s)}^{n}\right) d B_{u}^{H}\right|^{2 N}\right\} \\
:=B_{1}+B_{2}+B_{3} .
\end{gathered}
$$

It follows from the growth assumption in $\left(H_{1}\right)$, the estimate (49), and Hölder inequality that

$$
\begin{aligned}
B_{1} \leq & C_{N}(t-s)^{2 N-1} \\
& \times \int_{s}^{t} E^{W}\left|a\left(t, v_{n}(u), X_{v_{n}(s)}^{n}\right)\right|^{2 N} d u \\
\leq & R_{N}(t-s)^{2 N} .
\end{aligned}
$$

By Burkhölder-Davis-Gundy and Hölder inequalities and using the estimate (49), we obtain

$$
\begin{aligned}
B_{2} \leq & C_{N}(t-s)^{N-1} \\
& \times E^{W}\left[\int_{s}^{t}\left|b\left(t, v_{n}(u), X_{v_{n}(s)}^{n}\right)\right|^{2 N} d u\right] \\
\leq & R_{N}(t-s)^{N} .
\end{aligned}
$$


Finally, using the estimates (24) and (49), we get

$$
\begin{aligned}
B_{3} \leq & C_{N} E^{W} \Lambda_{\alpha}\left(B^{H}\right)^{2 N}(t-s)^{2 N(1-\alpha)+2 \alpha-1} \\
& \times \int_{s}^{t} \frac{\left\|c\left(t, v_{n}(u), X_{v_{n}(s)}^{n}\right)\right\|_{\alpha}^{2 N}}{(u-s)^{2 \alpha}} d u \\
\leq & R_{N}(t-s)^{2 N(1-\alpha)+2 \alpha-1} \\
& \times E^{W} \int_{s}^{t} \frac{1+\left\|X_{v_{n}(s)}^{n}\right\|_{\alpha}^{2 N}}{(u-s)^{2 \alpha}} d u \\
\leq & R_{N}(t-s)^{N} .
\end{aligned}
$$

Summing up, we deduce the desired result.

Let $P^{n}=P \circ X^{n}, n \geq 0$, be the sequence of probability measure induced by $X^{n}$ on $C_{0}^{\eta}$, then the sequence is tight (a similar result to Proposition 5.2 in Guerra and Nualart [9]).

Now, we can state that there exists a weak solution for (2), which follows the same computations to those given in Theorem 5.3 of Guerra and Nualart [9].

Theorem 9 (existence of weak solution). Suppose that the coefficients $a, b$, and $c$ satisfy the assumptions $\left(H_{1}\right),\left(H_{2}\right)$, and $\left(H_{3}\right)$ with $\beta>1-H, \delta>(1 / H)-1, \min \{\beta, \delta /(\delta+1)\}>1-\mu$, respectively. If $\max \{1-H, 1-\mu\}<\alpha<\min \{1 / 2, \beta, \delta /(\delta+1)\}$ and $\rho \leq 1 / \alpha$, then (2) has a unique weak solution.

Therefore, by the classical Yamada and Watanabe theorem $[17,18]$, Theorem 1 will be an immediate consequence of the pathwise uniqueness property and the existence of weak solutions (Theorem 9).

\section{Acknowledgments}

The authors would like to thank the referees for valuable comments and suggestions on this paper. The works was supported by the NSFC (11171062, 11201062, 40901241), the Innovation Program of Shanghai Municipal Education Commission (12ZZ063), the Research Project of Education of Zhejiang Province (Y201326507), the Natural Science Foundation of Zhejiang Province (Y5090377), the Key Natural Science Foundation of Anhui Educational Committee (KJ2013A133), Natural Science Foundation of Anhui Province (1308085QA14), and the Fundamental Research Funds for the Central Universities.

\section{References}

[1] F. Biagini, Y. Hu, B. Øksendal, and T. Zhang, Stochastic Calculus for Fractional Brownian Motion and Applications, Springer, London, UK, 2006.

[2] Y. Hu, "Integral transformations and anticipative calculus for fractional Brownian motions," Memoirs of the American Mathematical Society, vol. 175, no. 825, 2005.

[3] Y. S. Mishura, Stochastic Calculus for Fractional Brownian Motion and Related Processes, vol. 1929, Springer, Berlin, Germany, 2008.
[4] D. Nualart, The Malliavin Calculus and Related Topics, Springer, Berlin, Germany, 2nd edition, 2006.

[5] M. Zähle, "Integration with respect to fractal functions and stochastic calculus. I," Probability Theory and Related Fields, vol. 111, no. 3, pp. 333-374, 1998.

[6] L. Decreusefond and A. S. U. Üstünel, "Fractional Brownian motion: theory and applications," ESAIM Proceedings, vol. 5, pp. 75-86, 1998.

[7] K. Kubilius, "The existence and uniqueness of the solution of an integral equation driven by a $p$-semimartingale of special type," Stochastic Processes and Their Applications, vol. 98, no. 2, pp. 289-315, 2002.

[8] M. Zähle, "Stochastic differential equations with fractal noise," Mathematische Nachrichten, vol. 278, no. 9, pp. 1097-1106, 2005.

[9] J. Guerra and D. Nualart, "Stochastic differential equations driven by fractional Brownian motion and standard Brownian motion," Stochastic Analysis and Applications, vol. 26, no. 5, pp. 1053-1075, 2008.

[10] Y.S. Mishura and G. M. Shevchenko, "Existence and uniqueness of the solution of stochastic differential equation involving Wiener process and fractional Brownian motion with Hurst index $H>1 / 2$," Communications in Statistics, vol. 40, no. 1920, pp. 3492-3508, 2011.

[11] Y. Mishura and G. Shevchenko, "Mixed stochastic differential equations with long-range dependence: existence, uniqueness and convergence of solutions," Computers \& Mathematics with Applications, vol. 64, no. 10, pp. 3217-3227, 2012.

[12] E. Alòs and D. Nualart, "Anticipating stochastic Volterra equations," Stochastic Processes and Their Applications, vol. 72, no. 1, pp. 73-95, 1997.

[13] M. A. Berger and V. J. Mizel, "Volterra equations with Itô integrals. I," Journal of Integral Equations, vol. 2, no. 3, pp. 187245, 1980.

[14] M. A. Berger and V. J. Mizel, "Volterra equations with Itô integrals. II," Journal of Integral Equations, vol. 2, no. 4, pp. 319337, 1980

[15] M. Besalú and C. Rovira, "Stochastic Volterra equations driven by fractional Brownian motion with Hurst parameter $H>1 / 2$," Stochastics and Dynamics, vol. 12, no. 4, Article ID 1250004, pp. 1-24, 2012.

[16] P. Protter, "Volterra equations driven by semimartingales," The Annals of Probability, vol. 13, no. 2, pp. 519-530, 1985.

[17] T. Yamada and S. Watanabe, "On the uniqueness of solutions of stochastic differential equations," Journal of Mathematics of Kyoto University, vol. 11, pp. 155-167, 1971.

[18] S. Watanabe and T. Yamada, "On the uniqueness of solutions of stochastic differential equations. II," Journal of Mathematics of Kyoto University, vol. 11, pp. 553-563, 1971.

[19] D. Nualart and A. Răşcanu, "Differential equations driven by fractional Brownian motion," Collectanea Mathematica, vol. 53, no. 1, pp. 55-81, 2002.

[20] D. Hamadouche, "Invariance principles in Hölder spaces," Portugaliae Mathematica, vol. 57, no. 2, pp. 127-151, 2000. 


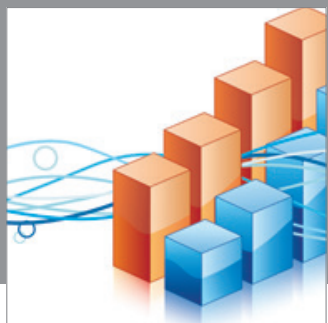

Advances in

Operations Research

mansans

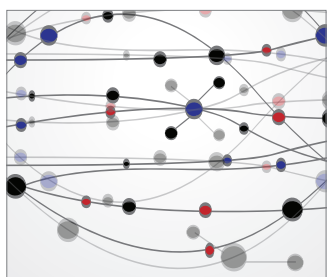

The Scientific World Journal
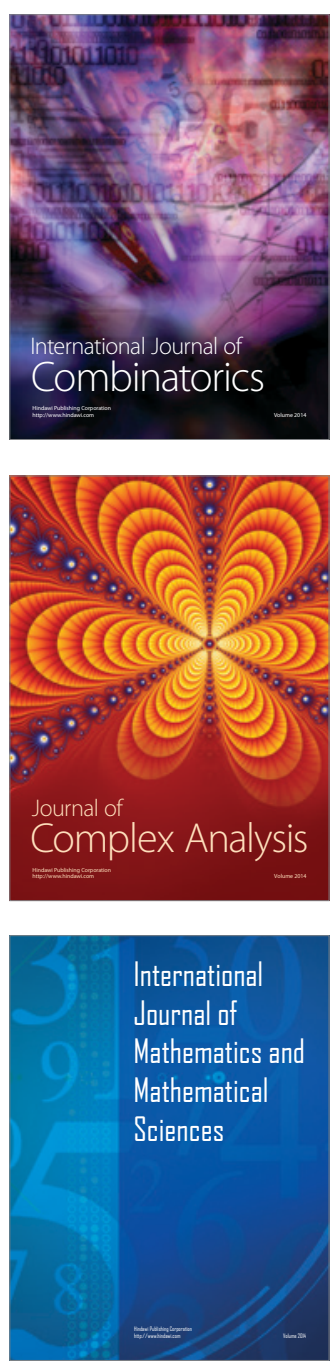
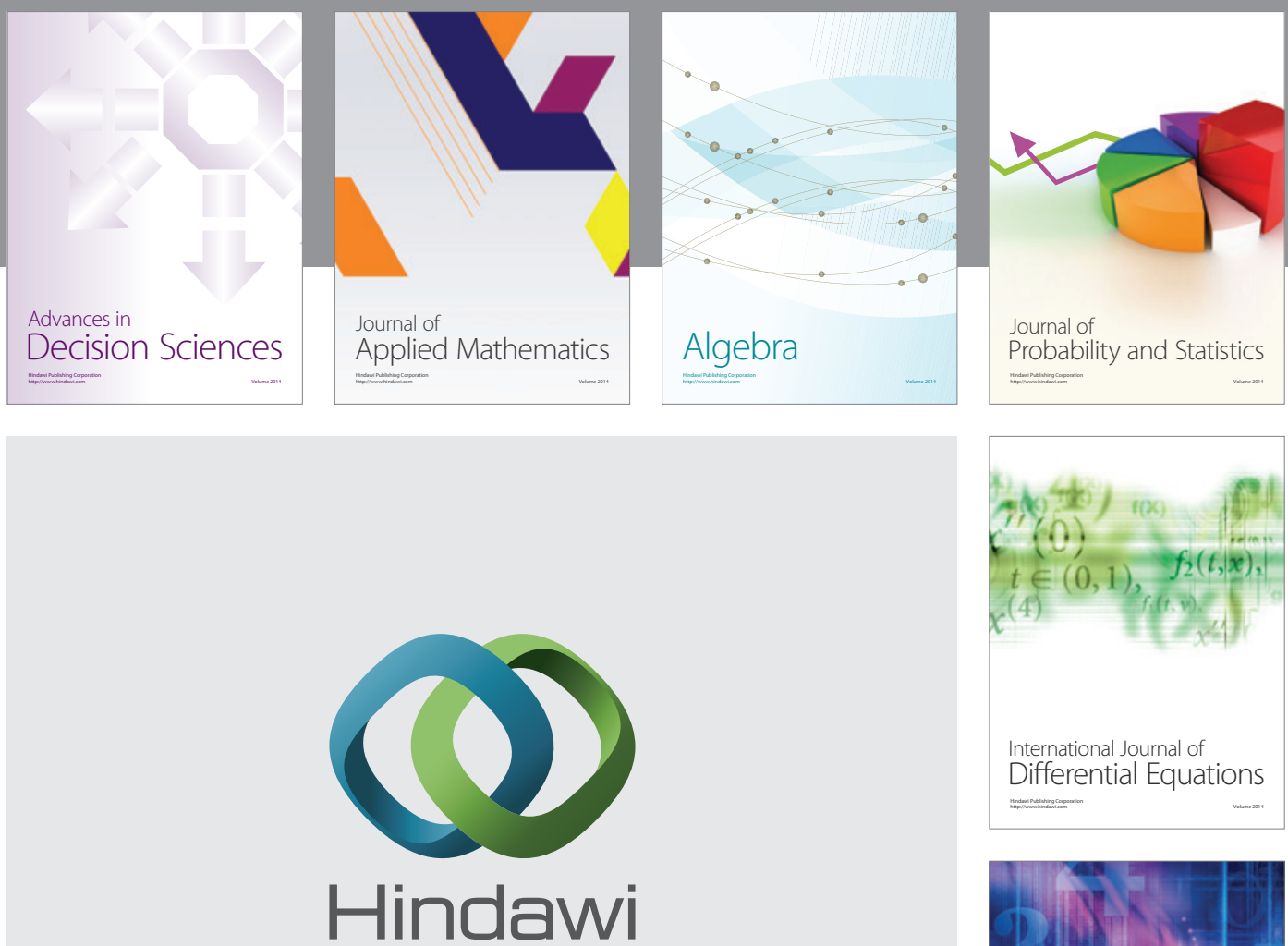

Submit your manuscripts at http://www.hindawi.com
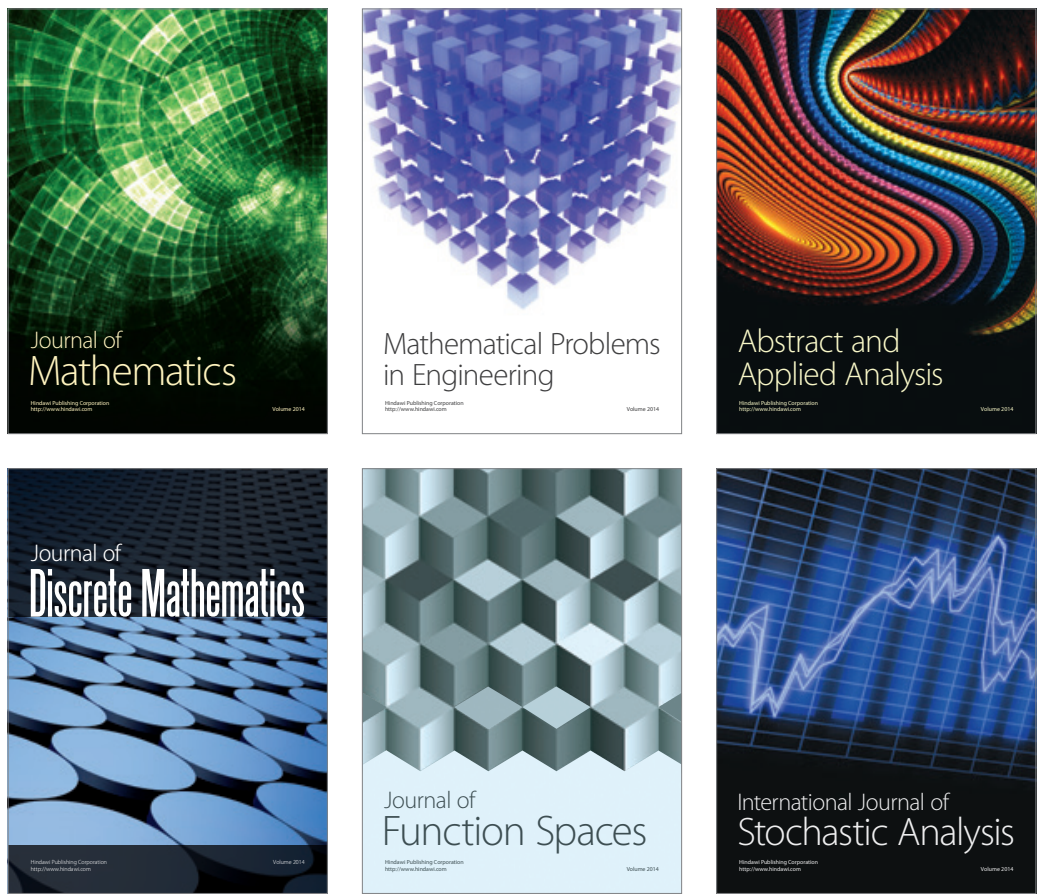

Journal of

Function Spaces

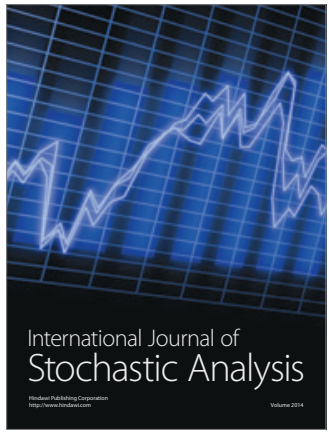

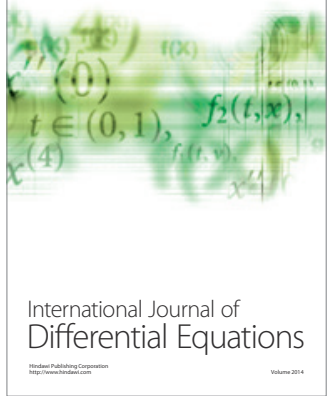
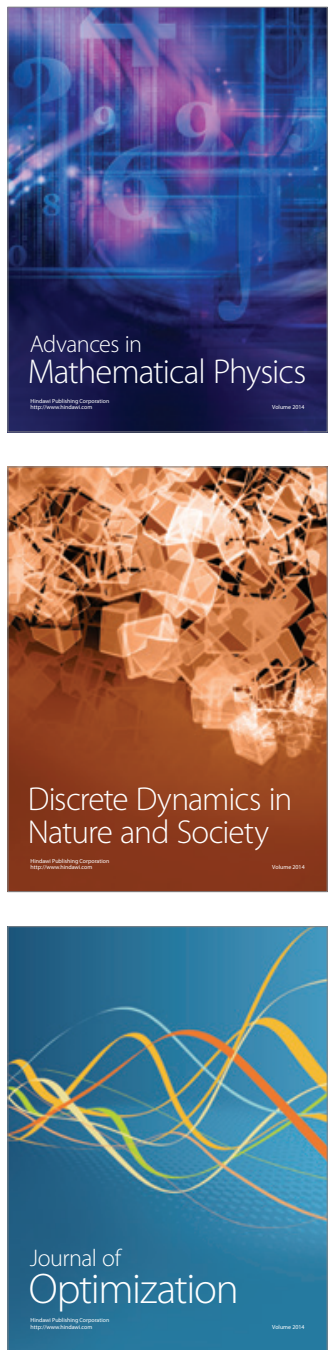